\title{
Utility of Syndromic Surveillance in Detecting Potential Human Exposures to Rabies
}

\author{
Kelley Bemis*, Megan T. Patel, Mabel Frias and Demian Christiansen \\ Communicable Disease, Cook County Department of Public Health, Forest Park, IL, USA
}

\section{Objective}

To determine whether unreported cases of potential human exposure to rabies can be detected using an emergency department (ED) syndromic surveillance system and to assess both reporting completeness and compliance with clinical guidelines related to rabies exposures in suburban Cook County.

\section{Introduction}

Rabies post-exposure prophylaxis (PEP) can prevent fatal encephalitis associated with exposure to the rabies virus. However, overuse and inappropriate administration of rabies PEP are common. ${ }^{1}$ Mandatory reporting of potential rabies exposures provides opportunities for public health practitioners to monitor the appropriateness of PEP administration and offer recommendations. In Illinois, potential human exposure to rabies, including any person started on PEP and any person with contact to a bat, must be reported to the local health authority. Previous investigations into the completeness of rabies reporting have concluded that active surveillance in addition to mandatory reporting may be useful. ${ }^{2}$ As rabies PEP is often given in an emergency department setting, syndromic surveillance records may provide a basis for estimating completeness of reporting and identifying candidates for active surveillance follow up.

\section{Methods}

Emergency department records from 45 local hospitals between $1 / 1 / 2013$ and 6/30/2015 were queried for chief complaints or discharge diagnoses pertaining to rabies, PEP, or contact with a bat. Exclusionary terms and manual record review eliminated unrelated visits. Cases of potential human exposure to rabies reported to the Cook County Department of Public Health (CCDPH) during the same time period were extracted from the Illinois National Electronic Disease Surveillance System. Cases were matched to ED records based on provider, visit date, age, sex, and zip code. The remaining unmatched individuals with $\geq 2$ visits were considered probable unreported instances of PEP initiation. Demographics of unreported individuals were compared to reported individuals using chi square.

\section{Results}

Between 1/1/2013 and 6/30/2015, 241 individuals visited local EDs with a chief complaint or discharge diagnosis related to bat contact or rabies PEP. Of these $241,63(26 \%)$ were previously reported to CCDPH. Of the remaining 178,80 (45\%) had $\geq 2$ visits suggesting a true instance of unreported PEP initiation. Reporting of these individuals was less common in winter compared to spring, summer, and fall (18\% versus $64 \%, 48 \%, 54 \%$, respectively, $\mathrm{p}=.03$ ). Region of patient residence also exhibited an association with reporting ranging from $92 \%$ in the South District to $28 \%$ in the North District $(\mathrm{p}<.01)$. Regional trends likely reflect differential reporting behaviors among hospitals in the area, ranging from $100 \%$ to $0 \%$. Of note, the 63 previously reported individuals identified by syndromic surveillance queries represented only $54 \%$ of the individuals reported to CCDPH during the same time period, suggesting that underreporting as measured here remains a significant underestimate. Based on these results, CCDPH instituted a new active surveillance policy for individuals visiting local EDs with a chief complaint or discharge diagnosis related to bat contact or rabies PEP, retroactive to $1 / 1 / 2015$. Individuals identified through active surveillance will be compared with individuals reported through passive surveillance to assess differences in whether PEP was given in accordance with recommendations and administered correctly.

\section{Conclusions}

A large proportion of potential human exposures to rabies in Suburban Cook County are not reported. Analysis of syndromic surveillance records is an effective tool for evaluating reporting completeness, identifying targets for active surveillance, and ensuring compliance with clinical best practice and reporting requirements.

\section{Keywords}

Syndromic surveillance; Rabies; Post-exposure prophylaxis; Evaluation

\section{References}

1. Moran GJ, Talan DA, Mower W, et al. Appropriateness of rabies postexposure prophylaxis treatment for animal exposures. JAMA. 2000 Aug 23;284(8):1001-7.

2. Thiede H, Close NS, Koepsell J, Baer A, Duchin JS. Completeness of reporting of rabies postexposure prophylaxis in King County, Washington. J Public Health Manag Pract. 2008 Oct;14(5):448-53.

\section{*Kelley Bemis}

E-mail: kbemis@cookcountyhhs.org 